\title{
190. Ridiological Evaluations of the Treatments of Whiplash Injury with Special Attention on Barre-Lieou's Syndrome
}

\author{
Yoshito KIRITA \\ Division of Orthopaedic Surgery, Tenri Hospital, Tenri City
}

Two hundreds and fifty patients with whiplash injury have been radiologically evaluated during the courses of treatment. Instabilities in the cervical vertebrae were more frequently observed among the patients injured before more than two weeks than the patients with fresh injury.

Special attention was paid for the evaluation of cervical instability of eight patients with Barré-Liéou's syndrome. It was noted that the instability between third and fourth cervical vertebrae would play an important role in the manifestation of the symptoms.

Anterior spine fusion between third and fourth, fourth and fifth or fifth and sixth cervical vertebrae has completely improved the symptoms.

\section{Trapezius Fasciectomy for "Whiplash Injury"}

\author{
Nobuyuki Tsuzukr \\ Orthopedic Surgery, Kanto Rosai Hospital
}

The persistent pain around the neck and the shoulder of chronic patients of "Whiplash Injury", which disappeared temporarily by procaine block, could be relieved by the resection of the corresonding part of the trapezius fascia in all of 13 subjects.

Moreover, the stiffness and the tenderness of the lateral cervical portion and the supraclavicular fossa disappeared after the fasciectomy of the ipsilateral suprascapular rgion. The dysesthesia of the arm of the same side also disappeared after that resection, but it reappeared 1 or 2 months later in all cases. Occipitalgia reappeared in one case 1 month later. Those pains which were regarded as rootor disc-origin remained unchanged.

Macroscopically, the fascial hypertrophy and adhesion to the muscle, and, microscopically, hypertrophy and hyperplasia of collagen fibers of the fascia, blood and lymph stasis, and muscle damages and fibrosis were observed.

The fascia, with or without primary lesion, might fall into degeneration chiefly because of circulatory disturbances and mechanical stimuli, and play a role in aggravating "Whiplash Injury." 